\title{
Air/Liquid Interfacial Nanoassembly of Molecular Building Blocks into Preferentially Oriented Porous Organic Nanosheet Crystals via Hydrogen Bonding
}

Rie Makiura, ${ }^{*}, \dagger, \S_{\odot}$ Kohei Tsuchiyama, ${ }^{\ddagger}$ Ehmke Pohl, ${ }^{\|}$Kosmas Prassides, ${ }^{\perp}$ Osami Sakata, ${ }^{\#}$ Hiroo Tajiri, ${ }^{\otimes}$ and Oleg Konovalov ${ }^{\nabla}$

${ }^{\dagger}$ Department of Materials Science, Graduate School of Engineering, Osaka Prefecture University, Osaka 599-8570, Japan

${ }^{\ddagger}$ Nanoscience and Nanotechnology Research Center, Research Organization for the 21st Century, Osaka Prefecture University, Osaka 599-8570, Japan

${ }^{\S}$ PRESTO, Japan Science and Technology Agency, Saitama 332-0012, Japan

"Department of Chemistry, Durham University, Durham DH1 3LE, U.K.

${ }^{\perp}$ WPI-Advanced Institute for Materials Research (WPI-AIMR), Tohoku University, Sendai 980-8577, Japan



${ }^{\otimes}$ Research and Utilization Division, Japan Synchrotron Radiation Research Institute (JASRI/SPring-8), Hyogo 679-5148, Japan

${ }^{\nabla}$ European Synchrotron Radiation Facility (ESRF), 38000 Grenoble, France

S Supporting Information

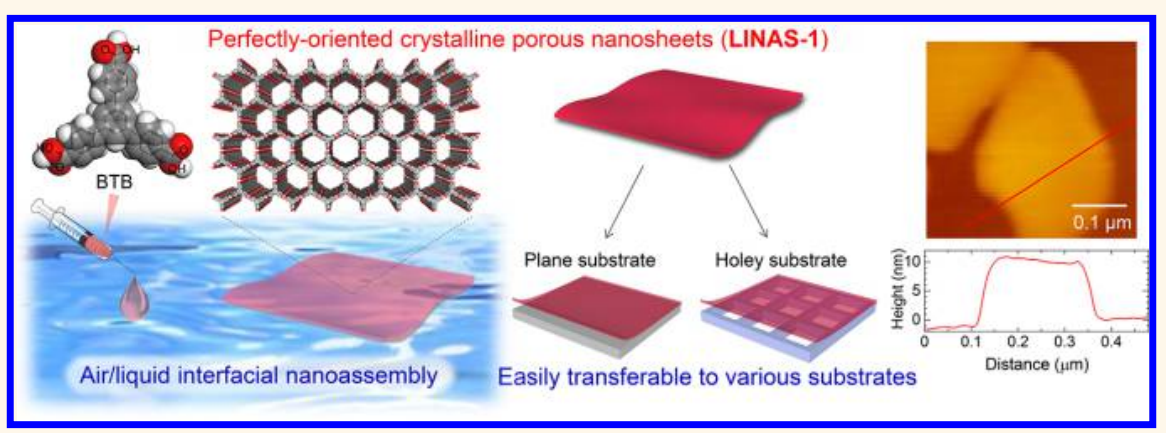

ABSTRACT: Nanosheets with highly regulated nanopores are ultimately thin functional materials for diverse applications including molecular separation and detection, catalysis, and energy conversion and storage. However, their availability has hitherto been restricted to layered parent materials, covalently bonded sheets, which are layered via relatively weak electrostatic interactions. Here, we report a rational bottom-up methodology that enables nanosheet creation beyond the layered systems. We employ the air/liquid interface to assemble a triphenylbenzene derivative into perfectly oriented highly crystalline noncovalent-bonded organic nanosheets under ambient conditions. Each molecular building unit connects laterally by hydrogen bonding, endowing the nanosheets with size- and position-regulated permanent nanoporosity, as established by in situ synchrotron X-ray surface crystallography and gas sorption measurements. Notably, the nanosheets are constructed specifically by interfacial synthesis, which suppresses the intrinsic complex interpenetrated structure of the bulk crystal. Moreover, they possess exceptional long-term and thermal stability and are easily transferrable to numerous substrates without loss of structural integrity. Our work shows the power of interfacial synthesis using a suitably chosen molecular component to create two-dimensional (2D) nanoassemblies not accessible by conventional bulk crystal exfoliation techniques.

KEYWORDS: nanosheet, air/liquid interface, porous material, membrane, self-assembly, two-dimensional material, oriented film

$\mathrm{N}$ anosheets - free-standing 2D nanomaterials - with long range structural order have been attracting considerable attention because of their intrinsic properties derived from their dimensionality and of their potential to be used as
Received: June 26, 2017

Accepted: September 21, 2017

Published: October 24, 2017 




Figure 1. Morphological and structural varieties of BTB depending on fabrication technique. (a) Triangular-shaped BTB molecules form hexagonal rings via hydrogen-bonded carboxylic acid dimers. Conventional bulk synthesis results in rodlike crystals with multiply interpenetrated rings. Interfacial synthesis controls the molecular orientation and prevents interpenetration, leading to perfectly oriented nanosheets with long-range molecular columns and size- and position-regulated nanopores. (b) Simple interfacial synthetic process involves spreading BTB solution on pure water subphase, resulting in the creation of crystalline porous nanosheets (LINAS-1) easily transferable to numerous substrates. (c) Contrasting micrometer-scale bulk BTB crystal. Rodlike objects similarly shaped to the bulk crystals are formed by conventional solution-based film fabrication techniques such as dip casting (d) and drop casting (e) on Si substrates. (f) Vacuum deposition on an Si substrate results in the formation of an amorphous film (Figure S9). The morphological images are obtained by AFM, (b, d-f) and optical microscopy (c), respectively.

ultrathin components in nanotechnological devices. ${ }^{1,2}$ Incorporation of highly regulated nanopores into the $2 \mathrm{D}$ structure leads to additional enormous capacity for diverse applications. ${ }^{3-5}$ Nanosheets so far are greatly restricted to layered or lamellar systems in which individual sheets are formed by strong interactions between atoms/molecules such as covalent or coordination bonds, and the sheets stack via relatively weak electrostatic interactions. ${ }^{6}$ By utilizing this hierarchy of interactions, layered bulk materials can be delaminated by chemical/physical treatments, leading to the isolation of nanosheets. ${ }^{7-9}$ Nanosheets with well-defined nanopores have been created with layered zeolites $^{10,11}$ and metal- and covalent-organic frameworks ${ }^{12,13}$ by the delamination process. Such top-down exfoliation not only limits the variety of materials but also restricts their use because it necessitates multiprocessing, which includes bulk synthesis, exfoliation, stabilization in solution, and deposition on a substrate, while it still remains difficult to place them uniformly on aimed positions. ${ }^{7}$ Although bottom-up approaches utilizing liquid interfaces for creating porous nanosheets have been reported, applicability is still limited to covalently bonded layered systems, including coordination networks. ${ }^{14-18}$ On the other hand, noncovalent bonds/interactions such as van der Waals interactions, dipolar coupling, and hydrogen bonding also play important roles for creating a variety of supramolecular assemblies, including $2 \mathrm{D}$ networks. ${ }^{19,20}$

Developing a rational methodology for creating nanosheets beyond such layered systems is a critical issue to be addressed that will further open the way for their use in a range of important applications. Indeed, there are various molecular-based functional solids, including biomaterials, which assemble by utilizing only electrostatic interactions such as hydrogen bonding and van der Waals forces. ${ }^{21,22}$ One of the important features of these 


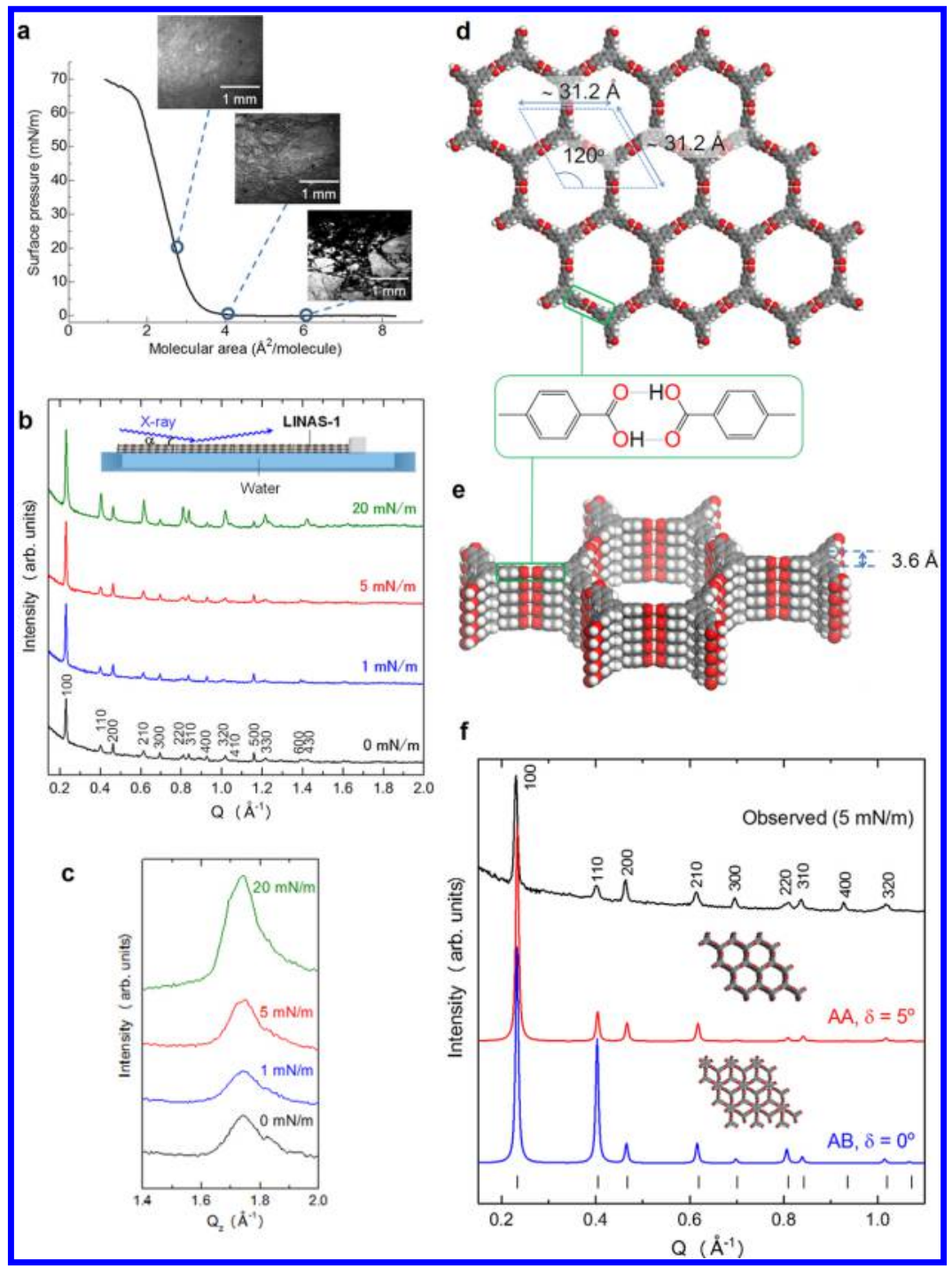

Figure 2. Structural characterization of LINAS-1 by in situ GIXRD measurements at the air/liquid interface. (a) Surface-pressure-meanmolecular-area ( $\Pi-A)$ isotherm for LINAS-1 with surface morphological images (inset) obtained by Brewster angle microscopy (BAM) at selected $\Pi$. (b, c) Observed in situ in-plane, (b) and out-of-plane, and (c) synchrotron GIXRD $(\lambda=1.549 \AA, Q=4 \pi \sin \theta / \lambda$, incident angle, $\alpha=0.12^{\circ}$ ) profiles at surface pressures, $\Pi=0,1,5$, and $20 \mathrm{mN} / \mathrm{m}$ together with schematic representation of the scattering geometry. (d) Basal plane projection and (e) stacking motif of the LINAS-1 crystalline structure derived from the GIXRD measurements. (f) Measured in situ (black line, $\Pi=5 \mathrm{mN} / \mathrm{m}$ ) and calculated (red line: AA slip-stacking with slip-angle, $\delta=5^{\circ}$; blue line: AB stacking model with neighboring honeycomb networks shifted horizontally by half the unit-cell size) in-plane synchrotron XRD profiles. Ticks mark the Bragg reflection positions. The tick marks show the reflection positions.

materials is that they can assemble under mild conditions without recourse to any high-temperature treatment. On the other hand, in forming stable structures, they tend to pack densely to maximize the attractive interactions and minimize empty space. Therefore, such materials tend to adopt nonporous structures. ${ }^{23}$ As a result, even for bulk systems, creating molecular-based porous frameworks is an ongoing target ${ }^{24-26}$ and obtaining such porous systems as nanosheets constitutes an even greater challenge.

Here, we present a quick operationally facile direct bottom-up procedure of assembling highly crystalline organic nanosheets without any intermolecular covalent bonds utilizing air/liquid interfaces under ambient conditions (Figure 1a top). The nanosheets are endowed with both size- and position-regulated nanopores and are completely oriented-the pore channels align vertically to the liquid surface. They are easily transferable to various substrates, including holey grids, while retaining their highly crystalline structure (Figure $1 \mathrm{~b}$ ). Notably, the crystal structure of the nanosheets differs from that of their equivalent bulk crystals, which adopt a multiply interpenetrated structure (Figure 1a (bottom),c). The highly crystalline porous 2D network is specifically realized at the air/liquid interface and is not accessible by conventional film fabrication techniques such as dip casting (Figure 1d), drop casting (Figure 1e), and vacuum deposition (Figure 1f, see the Methods). Our work shows the power of interfacial synthesis using molecular building blocks for creating 2D nanomaterials.

\section{RESULTS AND DISCUSSION}

Nanosheet Preparation. In order to assemble the porous organic nanosheets, we selected as a building block 1,3,5-tris-(4carboxyphenyl)-benzene (BTB), which possesses peripheral carboxylic acid linkers (Figure 1). Nanosheet (LINAS-1: liquidinterfacially assembled nanosheet no. 1) growth involved spreading 


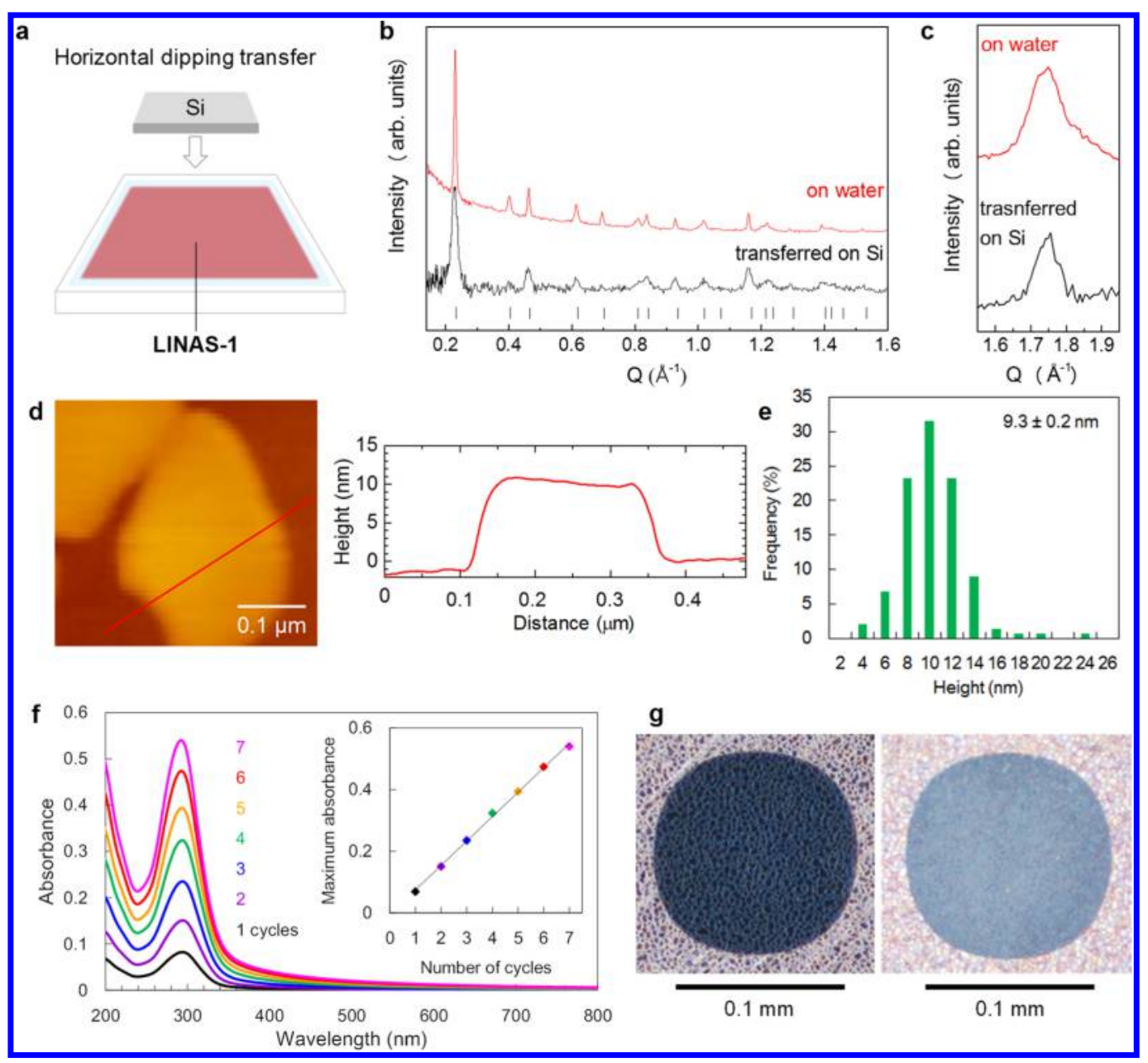

Figure 3. Integrity and morphological characterization of LINAS-1 after transfer onto solid substrates. (a) Schematic illustration of the horizontal dipping deposition method for nanosheet transfer from the liquid surface to the solid substrate surface. (b) In-plane and (c) out-of-plane synchrotron GIXRD profiles for LINAS-1 transferred on Si substrate (black line) together with those on water surface (red line). The tick marks show the reflection positions. (d) AFM image of LINAS-1 on Si and corresponding height profile along the track marked in red. (e) Height distribution of LINAS-1 evaluated from the AFM images. (f) Ultraviolet-visible (UV-vis) absorption spectra of LINAS-1 on a quartz substrate after successive cycles of sheet deposition. Inset: maximum absorbance versus number of sheet-deposition cycles. (g) Optical microscope image of a holey substrate with micromesh before (left) and after (right) LINAS-1 transfer.

of BTB solution in toluene/methanol onto a water subphase in a Langmuir trough at room temperature (Figure 1b). Nanosheet formation at the air/liquid surface was followed by monitoring the surface pressure-mean molecular area $(\Pi-A)$ isotherms (Figure 2a) and by Brewster angle microscopy (BAM) as the surface was compressed (Figure 2a (inset) and Figure S1).

Structural Characterization of LINAS-1 by in Situ GIXRD Measurements at the Air/Liquid Interface. Detailed insight into the LINAS-1 formation and its molecular order was obtained by in situ grazing-incidence synchrotron X-ray diffraction (GIXRD) measurements directly at the air/liquid interface. The observation of sharp clearly resolved Bragg peaks in the in-plane (sensitive to the lattice dimensions parallel to the interface) GIXRD profile of LINAS-1 at $\Pi=0 \mathrm{mN} / \mathrm{m}$ (Figure $2 \mathrm{~b}$ ) provides the signature that assembly of a large-scale highly crystalline structure occurs immediately upon spreading BTB onto the water surface. All peaks to a scattering vector, $Q_{x y}=2.1 \AA^{-1}$ index as $(h k 0)$ on a metrically hexagonal unit cell with in-plane lattice parameters, $a=b=31.17(1) \AA$. Out-of-plane GIXRD patterns (Figure 2c) reveal that LINAS-1 is also highly ordered in the direction vertical to the interface. Increasing $\Pi$ leads to an increase in peak intensity without a change in peak position for both in-plane and out-of-plane GIXRD measurements implying that surface compression does not affect the highly crystalline molecular organization but only the water surface coverage (Figure 2b,c).

Investigation of the crystalline LINAS-1 structure was initiated by considering as starting model the bulk BTB crystal structure, which we determined from single-crystal XRD data (Figures S2-S6 and Table S1). BTB crystallizes with a monoclinic structure comprising interpenetrated $2 \mathrm{D}$ honeycomb networks. Hexagonal networks of size, $a=b \sim 31.2 \AA$ are constructed by hydrogen-bonded carboxylic acid dimers (Figure 1a and Figure S5) and form columns via BTB $\pi-\pi$ stacking in an eclipsed-slipped arrangement (slip angle, $\delta=1.6-5.2^{\circ}$ ) at interlayer distances of 3.6-3.8 $\AA$ (Figure S6). Three- or four-BTB slabs align along different directions resulting in a complex interpenetrated framework structure (Figure 1a) comparable with those of other porous materials ${ }^{26}$ including BTB polymorphs. ${ }^{27}$

The LINAS-1 structural model (Figure 2d) incorporates the same 2D honeycomb motif of hydrogen-bond-linked BTB molecules. This is supported by the comparable in-plane lattice sizes of LINAS-1 and bulk BTB together with evidence from IR spectroscopy (Figure S7). However, the LINAS-1 in-plane XRD 


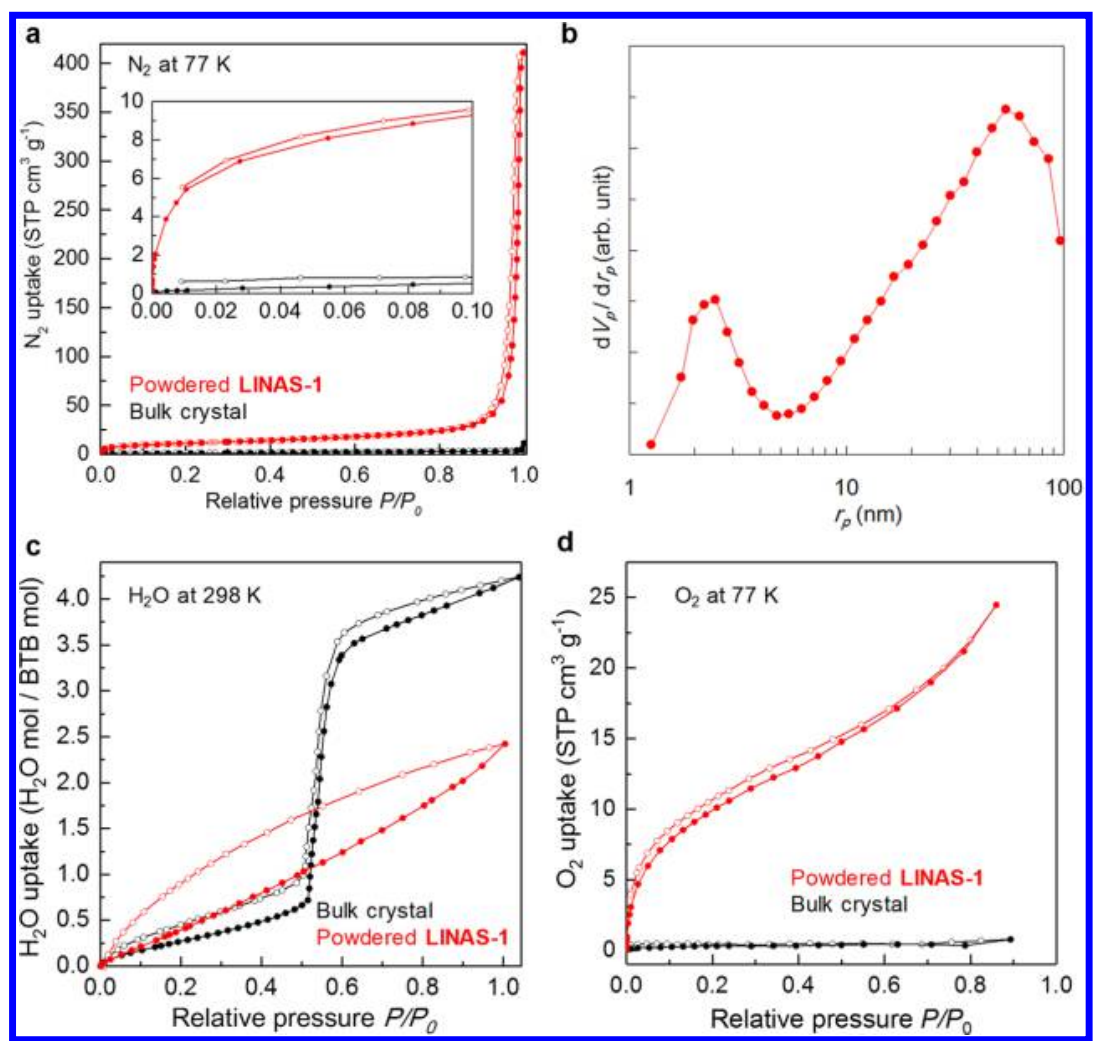

Figure 4. Gas-sorption properties of LINAS-1. (a) $\mathrm{N}_{2}$-sorption isotherms (77 K) for LINAS-1 (red) and bulk BTB crystals (black). Isotherms show both adsorption (dots) and desorption (open circles) data. Inset: expanded view of the low-pressure region. (b) Pore-size distribution curve for LINAS-1 obtained from Barrett-Joyner-Halenda (BJH) analysis of the $\mathbf{N}_{2}$-sorption isotherm. (c) Water vapor sorption isotherms $(298 \mathrm{~K})$. (d) $\mathrm{O}_{2}$-sorption isotherms $(77 \mathrm{~K})$.

profile is different from that of bulk BTB (Figures S8 and S9), and none of the Bragg reflections coincide with those measured in out-of-plane geometry (Figure $2 \mathrm{~b}$ ). This implies that LINAS-1 is free from the interpenetrated motif of bulk BTB and the honeycomb network plane is perfectly oriented parallel to the water surface. With the aid of the liquid interface as a crafting medium, exquisite control of the molecular orientation is achieved at the nanoscale and the formation of the complex interpenetrated structure is suppressed. The derived interlayer distance of $3.6 \AA$ - consistent with that obtained from single crystal XRD - also naturally points to the adoption of a $\pi-\pi$-stacked structure vertical to the water surface (Figure 2e). In-plane XRD profile simulations using eclipsed $\mathrm{AA}$ and staggered $\mathrm{AB}$ layerstacking modes clearly favor the former (Figures S10-S12). Allowing for a slip angle $\left(\delta=5^{\circ}\right)$ in the eclipsed stacking motif of the layers leads to best agreement with experiment (Figure 2f).

Integrity and Morphological Characterization of LINAS-1 after Transfer onto Solid Substrates. LINAS-1 is remarkably versatile and exceptionally robust. First, it can be easily transferred to a variety of solid substrate surfaces including holey supports (Figure 1a) and grow in a layer-by-layer manner by a horizontal dipping method (Figure 3a) without loss of structural integrity. This is immediately apparent by comparison of in-plane and out-of-plane synchrotron XRD patterns for LINAS-1 deposited on a $\mathrm{Si}(100)$ substrate and LINAS-1 at the air/liquid interface $(\Pi=5 \mathrm{mN} / \mathrm{m}$, Figure $3 \mathrm{~b}, \mathrm{c})$. There are no differences in position and relative intensity of the Bragg reflections (a change in peak width results from differences in instrumental resolution, see the Methods). The results confirm that the preferentially oriented highly crystalline LINAS-1 structure is retained intact after transfer on the Si substrate.
The sheet thickness and surface morphology of LINAS-1 transferred on $\mathrm{Si}$ were evaluated by atomic force microscopy (AFM) (Figure 3d). The height (thickness, Figure 3e) distribution reveals monodispersity with lateral dimension of the nanosheets (maximum Feret diameter, Figure S13) of $300 \pm 10 \mathrm{~nm}$. In particular, LINAS-1 has excellent height uniformity with average height of 9.3(2) nm, implying an aspect ratio greater than 30 , in good agreement with the crystalline domain size, $\sim 9 \mathrm{~nm}$ estimated from the full-width-at-half-maximum (fwhm) of the out-of-plane peak (Figure 3c) using Scherrer's equation. Given the interlayer distance of $3.6 \AA$, this implies that $\sim 25 \mathrm{BTB}$ molecules stack along the out-of-plane direction uniformly throughout the nanosheet. The Feret diameter also compares well with the lateral crystalline domain size $(\sim 150 \mathrm{~nm})$ estimated from the in-plane GIXRD results. This implies that each nanosheet observed in the AFM measurements corresponds to a single crystalline domain. A red-shifted peak observed in the photoluminescence (PL) spectrum of LINAS-1 supports the longrange stacking order compared to the evaporated amorphous film and the bulk crystal in which BTB stacking is limited to four molecules (Figure S14).

Although there are various reports on ordered molecular assemblies, including BTB, on solid substrates, ${ }^{28,29}$ the resulting molecular arrangements are highly dependent on substrate features. In order to obtain the desired structures, selection of substrate materials is restricted or surface treatment is necessary, sometimes leading to sacrifice function. Here, we also confirmed that successful transfer of LINAS-1 (Figure S15) and sequential layer-by-layer growth by repeated deposition (Figure $3 \mathrm{f}$ and Figure S16) can be made to numerous other substrates besides $\mathrm{Si}$, such as quartz, gold, indium-tin oxide, and graphite. This is a 
significant merit of our methodology as the assembled networks can be utilized on desired substrates without suffering from any structural changes. For example, nanosheets obtained by deposition on holey substrates can be used as separation membranes, while those transferred onto electrode substrates can be integrated in thin-film devices such as thin-film transistors and sensors. As shown in Figure 1, the perfectly oriented honeycomb network of LINAS-1 cannot be created on Si substrates by other techniques such as dip casting (Figure 1d), drop casting (Figure 1e), and vacuum deposition (Figure 1f). Similar honeycomb networks of BTB have been observed by scanning tunneling microscopy (STM), ${ }^{30-33}$ but only on specific atomically flat conductive substrates such as graphite, $\mathrm{Au}(111)$, and $\mathrm{Ag}(111)$. Moreover, the thickness is restricted to that of a monolayer, mainly for the aim to obtain clear images by STM measurements. This contrasts with the fine-tuning of the sheet thickness achieved by our technique. Linear increase of the absorbance with increasing number of deposition cycles (Figure $3 \mathrm{f}$ (inset)) provides unambiguous evidence that each deposition step leads to layering of uniform nanosheets with retention of the preferentially oriented structure of multiply deposited LINAS-1, confirmed by GIXRD (Figures S17 and S18). Such layer-by-layer growth allows exquisite tuning of film thickness at the nanoscale.

LINAS-1 is also stable as a free-standing film formed through suspension on a micromesh holey-grid support (Figure $3 \mathrm{~g}$ ). Powdered LINAS-1 can be prepared in large quantities by filtration using a membrane filter-the XRD profile is identical to that of LINAS-1 on Si (Figure S19), again confirming that the porous crystalline network survives even in the absence of any surface support. Finally, LINAS-1 is endowed with exceptional stability to both heating and aging. Both in situ and ex situ XRD measurements and thermogravimetric and differential thermal analysis (TG-DTA) reveal that the perfectly oriented crystalline structure of Si-deposited LINAS-1 is thermally stable to $\sim 20{ }^{\circ} \mathrm{C}$ despite the nanometer-scale thickness of the sheets (Figures S23 and S24). Notably, an exothermic peak corresponding to the structural change observed by diffraction is evident in the DTA curve of LINAS-1 at $230^{\circ} \mathrm{C}$ - this peak is also observed for the BTB bulk crystals, but at a lower temperature $\left(187^{\circ} \mathrm{C}\right)$. This implies that the structure with perfect molecular orientation in LINAS-1 is more stable than that of the bulk crystal with nonoriented interpenetrated motif. Similarly, GIXRD profiles of LINAS-1 on Si kept in air for three years are identical to those of the same as-prepared sample (Figure S21) establishing its extraordinary stability to long-term aging in the atmosphere.

Gas Sorption Characteristics. The LINAS-1 porosity was evaluated by $\mathrm{N}_{2}$-sorption isotherm measurements at $77 \mathrm{~K}$ (Figure 4a). Bulk BTB crystals show an IUPAC type-III shaped isotherm, ${ }^{34}$ characteristic of a closely packed structure in agreement with the crystal structure determination. On the other hand, the LINAS-1 isotherm exhibits a combined type-I/type-IV shape revealing the presence of both micro/meso- and macropores in LINAS-1. The sharp increase in $\mathrm{N}_{2}$ uptake at the lowpressure region $\left(P / P_{0}=0-0.02\right)$ implies that the $\mathrm{N}_{2}$ molecules are introduced into the micro/mesopores of the nanosheet. The micro/mesopore radii, $r_{\mathrm{p}}$, extracted from Barrett-Joyner-Halenda (BJH) analysis ${ }^{35}$ (see the Methods) of the $\mathrm{N}_{2}$-adsorption isotherm range between 1 and $3 \mathrm{~nm}$ (Figure $4 \mathrm{~b}$ ), in agreement with the effective pore size of $\sim 2.7 \mathrm{~nm}$ of the proposed structural model (Figure $2 \mathrm{~d}$ ). The second sharp increase in $\mathrm{N}_{2}$ uptake at high relative pressures $\left(P / P_{0}=0.96-1.00\right)$ is characteristic of "house-of-cards" inter-nanosheet porosity, commonly present in sheet-shaped materials; the wide distribution in pore radii with a peak maximum at $54 \mathrm{~nm}$ in the $\mathrm{BJH}$ plot (Figure $4 \mathrm{~b}$ ) reflects the "house-of-cards" porosity.

Complementary water-vapor sorption measurements of LINAS-1 at $298 \mathrm{~K}$ reveal the absence of any sharp increase in water uptake (Figure 4c), implying lack of hydrophilic sites in the honeycomb network. The behavior contrasts markedly with that of bulk BTB, which shows a sharp increase in water vapor sorption. Even though the $\mathrm{N}_{2}$-sorption isotherm of the BTB bulk is consistent with a closely packed structure, the crystal possesses small penetrable cavities along the $\langle 101\rangle$ direction with a diameter of $0.27-0.28 \mathrm{~nm}$ (Figure S6), which exactly match the kinetic diameter of water molecules $(2.65 \AA)$ but are smaller than that of $\mathrm{N}_{2}(3.64 \AA)$. We consider that free carboxylic groups in the BTB bulk crystal surface attract the water molecules and guide them into the pore cavities. On the other hand, LINAS-1 has neither such effective hydrophilic free carboxylic sites in the open-pore direction nor size-matched pores to water molecules, resulting in featureless water-sorption behavior. This also attests to the high quality and porosity in LINAS-1.

We also examined $\mathrm{O}_{2}$ gas sorption of LINAS-1 at $77 \mathrm{~K}$ (Figure $4 \mathrm{~d}$ ). The isotherm in bulk BTB crystals is flat throughout the measured pressure region up to the relative pressure of 0.9 . In contrast, we observe a sharp increase in $\mathrm{O}_{2}$ uptake at the lowpressure region in the LINAS-1 isotherm that implies strong interaction between $\mathrm{O}_{2}$ molecules and LINAS-1 frameworks. We consider that in this case nonpolar $\mathrm{O}_{2}$ molecules preferably attach to the hydrophobic pore inner surfaces. This also supports the formation of the high quality nanosheets with nanoporosity $(\sim 2.7 \mathrm{~nm})$ large enough to accommodate $\mathrm{O}_{2}$ molecules (kinetic diameter, $3.46 \AA$ ) in contrast to the bulk material in which the cavity diameter is only $0.27-0.28 \mathrm{~nm}$.

\section{CONCLUSIONS}

The results presented here show the first successful construction of a single-crystal-like noncovalent organic framework nanosheet (named LINAS-1) by facile interfacial synthesis using molecular building blocks. In situ synchrotron X-ray diffraction at the air/ liquid interface established the immediate formation of highly crystalline LINAS-1 through the spontaneous organization of the molecular components upon their spreading onto the water surface at room temperature. LINAS-1 is completely oriented; the $2 \mathrm{D}$ honeycomb networks are parallel to and the $\pi-\pi$-stacked molecular columns are vertical to the water surface, while the corresponding bulk crystal adopts a nonoriented multiply interpenetrated structure. Such interpenetration is often seen in large framework materials, in which different sublattices occupy the same space resulting in the minimization of the void volume and the collapse of the porous motif. By virtue of using a liquid interface as an assembly work-platform, both the molecular orientation of the building components and the growth direction are finely controlled, suppressing the formation of intrinsically nonporous complex interpenetrated structures. The existence of both size- and position-regulated permanent effective pores was demonstrated by gas-sorption measurements.

LINAS-1 nanosheets display additional superior quality characteristics. They are exceptionally robust to both heating and environmental aging and are remarkably versatile; they can be transferred to diverse types of substrates such as silicon, quartz, gold, graphite, and holey grids while preserving their highly crystalline perfectly oriented structure intact. The versatility of the interfacial synthetic strategy opens the way for designing-in functional features to nanosheets derived from classes of materials 
that are not restricted to those with the potential for exfoliation of their bulk crystalline layered structures.

\section{METHODS}

Nanosheet Preparation (LINAS-1). A PTFE-coated Langmuir trough was filled with pure water or acidic water as a subphase. The surface of the subphase was carefully cleaned by mild surface-touch vacuuming. A $1 \mathrm{mM}$ solution of BTB in mixed toluene/methanol solvent $(2: 1, \mathrm{v} / \mathrm{v})$ was spread onto the water subphase with a microsyringe. Surface pressure-area $(\Pi-A)$ isotherm measurements were performed at a continuous barrier pressing speed of $500 \mu \mathrm{m} / \mathrm{s}$ at room temperature. The surface pressure was measured with the Wilhelmy plate method. Rapid increase of the surface pressure under surface compression implies formation of a BTB nanosheet at the air/liquid interface (LINAS-1).

Deposition of LINAS-1 on Solid Substrates. A horizontal dipping method was employed for the deposition of LINAS-1 on solid substrates such as quartz, $\mathrm{Si}, \mathrm{Au} / \mathrm{Cr} / \mathrm{Si}, \mathrm{HOPG}$, and microgrid. The substrate with LINAS-1 deposited was then rinsed with flowing distilled water, immersed in distilled water for $3 \mathrm{~min}$, and finally dried by blowing nitrogen. To stack additional sheets, the sheet transfer and rinse/ immerse/dry cycle was repeated.

Powdered LINAS-1. In order to prepare a sufficiently large amount of LINAS-1 for $\mathrm{N}_{2}$ and water vapor sorption measurements, the $1 \mathrm{mM}$ BTB solution in mixed toluene/methanol solvent $(2: 1, \mathrm{v} / \mathrm{v})$ was spread on the water subphase in a glass beaker. The LINAS-1 formed on the water subphase was then collected by filtering and the process of LINAS-1 formation, followed by filtration was repeated many times. A total of about $30 \mathrm{mg}$ of the powdered LINAS-1 was obstained. Of these, $24 \mathrm{mg}$ was used for the gas sorption measurements and $5 \mathrm{mg}$ for the powder XRD measurements.

In Situ Grazing-Incidence (GI) Synchrotron X-ray Diffraction (GIXRD) Measurements at Air/Liquid Interfaces. In situ GI synchrotron XRD measurements of LINAS-1 at the air/liquid interface were performed at room temperature with the six-circle diffractometer on beamline ID10B $(E=8.003 \mathrm{keV}, \lambda=1.549 \AA)$ at the ESRF (Grenoble, France). The dedicated PTFE Langmuir trough $(460 \times$ $170 \times 5 \mathrm{~mm}^{3}$ ) mounted on the diffractometer was equipped with a single movable barrier for film compression. LINAS-1 was prepared as described above. The surface pressure was kept constant during individual GIXRD measurements. The Langmuir trough was mounted on an active antivibration system and was enclosed inside an airtight acrylic case with polyimide windows. Water-saturated helium gas was introduced into the case. The incidence angle, $\alpha$, for the GIXRD measurements was set at $0.12^{\circ}$. The scattered X-rays were recorded by a onedimensional (1D) gas-filled position-sensitive detector with vertically located counting wires (VANTEC). The in-plane XRD profiles were collected by scanning over the in-plane $\theta$ angle, and the vertical (out-ofplane) scattered intensity was recorded at each $2 \theta$ angle. In order to improve the $2 \theta$ resolution in the in-plane direction and reduce background contribution, a Soller collimator $\left(0.08^{\circ}\right)$ was placed in front of the 1D detector. The average sheet domain size was estimated with Scherrer's equation. For the out-of-plane XRD measurements, the incident angle, $\alpha$, was also fixed at $0.12^{\circ}$ and the scattered X-rays were recorded by scanning over the exit out-of-plane $\theta$ angle.

Synchrotron XRD Measurements on Solid Substrates. Synchrotron XRD data of LINAS-1 transferred on Si substrates were collected at room temperature with a multiaxis diffractometer at beamline $\mathrm{BL}_{13 \mathrm{XU}^{36}}(\lambda=1.542$ or $1.548 \AA)$ in SPring-8. Helium gas was supplied through the cell during the measurements. Each data set was recorded using a scintillation counter. The GIXRD measurements were carried out at $\alpha=0.155^{\circ}$. Diffraction from the sample surface was observed in the in-plane direction (Figure 3B, background subtracted) with the same detection angle as $\alpha$. A Soller collimator $\left(0.4^{\circ}\right)$ was placed in front of the detector. The out-of-plane scans were carried out in $\theta-2 \theta$ scattering geometry (Figure $3 \mathrm{C}$ ).

Atomic Force Microscopy. AFM images were obtained with a $\mathrm{Si}$ cantilever $(15 \mathrm{~N} / \mathrm{m})$ on an SII SPA400 system at room temperature. Dynamic force mode was applied. The height and lateral diameter analysis was conducted on measurements from more than 140 domains in the AFM images.

Gas Sorption Isotherm Measurements. $\mathrm{N}_{2}, \mathrm{O}_{2}$, and $\mathrm{H}_{2} \mathrm{O}$ vapor sorption isotherm measurements of powdered LINAS-1 and of bulk BTB crystals were performed using a BELSORP-max automatic volumetric adsorption system (Bel Japan, Inc.) at 77, 77, and $298 \mathrm{~K}$, respectively. The samples were placed in a glass tube and dried under vacuum at $80{ }^{\circ} \mathrm{C}$ for $3 \mathrm{~h}$ (powdered LINAS-1) or at $150{ }^{\circ} \mathrm{C}$ for $24 \mathrm{~h}$ (bulk BTB) for solvent removal. The glass tubes containing the samples were set on the measurement system and heated under high vacuum (below $10^{-3} \mathrm{~Pa}$ ) at $80^{\circ} \mathrm{C}$ for $1 \mathrm{~h}$ prior to the measurements. The sample tubes were then introduced into a liquid nitrogen cryostat for the $\mathrm{N}_{2}$ and $\mathrm{O}_{2}$ sorption or a thermostat bath for the $\mathrm{H}_{2} \mathrm{O}$ vapor sorption measurements. Pressure changes were monitored and converted to $\mathrm{N}_{2}$ or $\mathrm{H}_{2} \mathrm{O}$ vapor uptake.

The pore radii, $r_{\mathrm{p}}$, were extracted from Barrett-Joyner-Halenda (BJH) analysis of the $\mathrm{N}_{2}$ absorption isotherm. The pore distribution curve is obtained by plotting the pore volume change ratio, $\mathrm{d} V_{\mathrm{p}} / \mathrm{d} r_{\mathrm{p}}$ versus pore radius. The $\mathrm{BJH}$ analysis is generally applied for cylindershaped mesopore-containing materials.

\section{ASSOCIATED CONTENT}

\section{Supporting Information}

The Supporting Information is available free of charge on the ACS Publications website at DOI: 10.1021/acsnano.7b04447.

Experimental details; bulk single crystal XRD analysis data; UV-vis and IR absorption spectra, PL spectra, powder XRD data, AFM and SEM images (PDF)

\section{AUTHOR INFORMATION}

\section{Corresponding Author}

*E-mail: r-makiura@mtr.osakafu-u.ac.jp.

ORCID 웅

Rie Makiura: 0000-0003-2589-0698

Osami Sakata: 0000-0003-2626-0161

\section{Notes}

The authors declare no competing financial interest.

The X-ray crystal structure of BTB is deposited in the Cambridge Crystallographic Data Centre (CCDC 1453166).

\section{ACKNOWLEDGMENTS}

We acknowledge financial support from the Japan Science and Technology Agency (JST) on "Precursory Research for Embryonic Science and Technology (PRESTO)” for a project of "Molecular technology and creation of new functions (JPMJPR12K8)", CREST, the Japan Society for the Promotion of Science (JSPS) on "Grants-in-Aid for Scientific Research, KAKENHI” (JP22108524, JP24108735 “coordination programming”, JP16H05968, JP16K13610, JP17H05139 “ $\pi$-system figuration"), MEXT "SCF for Promoting Science and Technology", the Kao Foundation for Arts and Sciences, the INAMORI foundation, the Japan Prize Foundation, Shiseido Female Researcher Science Grant, the Murata Science Foundation, and the Royal Society "International Exchange Scheme". The synchrotron X-ray radiation experiments were performed at the BL13XU beamline, SPring-8 (2013A1668) and at the ID10B beamline, ESRF. We thank Rigaku for the in situ GIXRD measurements upon heating and Y. Susami, Y. Shibata, and Y. Takabayashi for experimental support.

\section{REFERENCES}

(1) Bonaccorso, F.; Colombo, L.; Yu, G.; Stoller, M.; Tozzini, V.; Ferrari, A. C.; Ruoff, R. S.; Pellegrini, V. Graphene, Related Two- 
Dimensional Crystals, and Hybrid Systems for Energy Conversion and Storage. Science 2015, 347, 1246501.

(2) Seiki, N.; Shoji, Y.; Kajitani, T.; Ishiwari, F.; Kosaka, A.; Hikima, T.; Takata, M.; Someya, T.; Fukushima, T. Rational Synthesis of Organic Thin Films with Exceptional Long-Range Structural Integrity. Science 2015, 348, 1122-1126.

(3) Corma, A.; Fornes, V.; Pergher, S. B.; Maesen, T. L. M.; Buglass, J. G. Delaminated Zeolite Precursors as Selective Acidic Catalysts. Nature 1998, 396, 353-356.

(4) Zhang, J.; Yu, J.; Zhang, Y.; Li, Q.; Gong, J. R. Visible Light Photocatalytic $\mathrm{H}_{2}$-Production Activity of $\mathrm{CuS} / \mathrm{ZnS}$ Porous Nanosheets Based on Photoinduced Interfacial Charge Transfer. Nano Lett. 2011, 11, 4774-4779.

(5) Hatton, B.; Mishchenko, L.; Davis, S.; Sandhage, K. H.; Aizenberg, J. Assembly of Large-Area, Highly Ordered, Crack-Free Inverse Opal Films. Proc. Natl. Acad. Sci. U. S. A. 2010, 107, 10354-10359.

(6) Mas-Ballesté, R.; Gómez-Navarro, C.; Gómez-Herrero, J.; Zamora, F. 2D Materials: to Graphene and Beyond. Nanoscale 2011, 3, 20-30.

(7) Coleman, J. N.; Lotya, M.; O’Neill, A.; Bergin, S. D.; King, P. J.; Khan, U.; Young, K.; Gaucher, A.; De, S.; Smith, R. J.; Shvets, I. V.; Arora, S. K.; Stanton, G.; Kim, H. Y.; Lee, K.; Kim, G. T.; Duesberg, G. S.; Hallam, T.; Boland, J. J.; Wang, J. J.; et al. Two-Dimensional Nanosheets Produced by Liquid Exfoliation of Layered Materials. Science 2011, 331, 568-571.

(8) Kory, M. J.; Wörle, M.; Weber, T.; Payamyar, P.; van de Poll, S. W.; Dshemuchadse, J.; Trapp, N.; Schlüter, A. D. Gram-Scale Synthesis of Two-Dimensional Polymer Crystals and their Structure Analysis by XRay Diffraction. Nat. Chem. 2014, 6, 779-784.

(9) Kissel, P.; Murray, D. J.; Wulftange, W. J.; Catalano, V. J.; King, B. T. A Nanoporous Two-Dimensional Polymer by Single-Crystal-toSingle-Crystal Photopolymerization. Nat. Chem. 2014, 6, 774-778.

(10) Lai, Z.; Bonilla, G.; Diaz, L.; Nery, J. G.; Sujaoti, K.; Amat, M. A.; Kokkoli, E.; Terasaki, O.; Thompson, R. W.; Tsapatsis, M.; Vlachos, D. G. Microstructural Optimization of a Zeolite Membrane for Organic Vapor Separation. Science 2003, 300, 456-460.

(11) Varoon, K.; Zhang, X.; Elyassi, B.; Brewer, D. D.; Gettel, M.; Kumar, S.; Lee, J. A.; Maheshwari, S.; Mittal, A.; Sung, C. Y.; Cococcioni, M.; Francis, L. F.; McCormick, A. V.; Mkhoyan, K. A.; Tsapatsis, M. Dispersible Exfoliated Zeolite Nanosheets and their Application as a Selective Membrane. Science 2011, 334, 72-75.

(12) Peng, Y.; Li, Y.; Ban, Y.; Jin, H.; Jiao, W.; Liu, X.; Yang, W. MetalOrganic Framework Nanosheets as Building Blocks for Molecular Sieving Membranes. Science 2014, 346, 1356-1359.

(13) Chandra, S.; Kandambeth, S.; Biswal, B. P.; Lukose, B.; Kunjir, S. M.; Chaudhary, M.; Babarao, R.; Heine, T.; Banerjee, R. Chemically Stable Multilayered Covalent Organic Nanosheets from Covalent Organic Frameworks via Mechanical Delamination. J. Am. Chem. Soc. 2013, 135, 17853-17861.

(14) Makiura, R.; Motoyama, S.; Umemura, Y.; Yamanaka, H.; Sakata, O.; Kitagawa, H. Surface Nano-Architecture of a Metal-Organic Framework. Nat. Mater. 2010, 9, 565.

(15) Makiura, R; Konovalov, O. Interfacial Growth of Large-Area Single-Layer Metal-Organic Framework Nanosheets. Sci. Rep. 2013, 3, 2506.

(16) Makiura, R.; Usui, R.; Sakai, Y.; Nomoto, A.; Ogawa, A.; Sakata, O.; Fujiwara, A. Towards Rational Modulation of In-Plane Molecular Arrangements in Metal-Organic Framework Nanosheets. ChemPlusChem 2014, 79, 1352-1360.

(17) Murray, D. J.; Patterson, D. D.; Payamyar, P.; Bhola, R.; Song, W.; Lackinger, M.; Schlüter, A. D.; King, B. T. Large Area Synthesis of a Nanoporous Two-Dimensional Polymer at the Air/Water Interface. J. Am. Chem. Soc. 2015, 137, 3450-3453.

(18) Rodenas, T.; Luz, I.; Prieto, G.; Seoane, B.; Miro, H.; Corma, A.; Kapteijn, F.; Xamena, F. X. L.; Gascon, J. Metal-Organic Framework Nanosheets in Polymer Composite Materials for Gas Separation. Nat. Mater. 2015, 14, 48-55.

(19) Theobald, J. A.; Oxtoby, N. S.; Phillips, M. A.; Champness, N. R.; Beton, P. H. Controlling Molecular Deposition and Layer Structure with Supramolecular Surface Assemblies. Nature 2003, 424, 1029-1031.
(20) Elemans, J. A. A. W.; Lei, S.; De Feyter, S. Molecular and Supramolecular Networks on Surfaces: From Two-Dimensional Crystal Engineering to Reactivity. Angew. Chem. Int. Ed. 2009, 48, 7298-7332.

(21) Moulton, B.; Zaworotko, M. J. From Molecules to Crystal Engineering: Supramolecular Isomerism and Polymorphism in Network Solids. Chem. Rev. 2001, 101, 1629.

(22) Mannige, R. V.; Haxton, T. K.; Proulx, C.; Robertson, E. J.; Battigelli, A.; Butterfoss, G. L.; Zuckermann, R. N.; Whitelam, S. Peptoid Nanosheets Exhibit a New Secondary-Structure Motif. Nature 2015, $526,415-420$.

(23) Dunitz, J. D.; Gavezzotti, A. How Molecules Stick Together in Organic Crystals: Weak Intermolecular Interactions. Chem. Soc. Rev. 2009, 38, 2622-2633.

(24) McKeown, N. B. Nanoporous Molecular Crystals. J. Mater. Chem. 2010, 20, 10588-10597.

(25) Chen, T.-H.; Popov, I.; Kaveevivitchai, W.; Chuang, Y. C.; Chen, Y. S.; Daugulis, O.; Jacobson, A. J.; Miljanić, O. Š. Thermally Robust and Porous Noncovalent Organic Framework with High Affinity for Fluorocarbons and CFCs. Nat. Commun. 2014, 5, 5131.

(26) Chen, B.; Eddaoudi, M.; Hyde, S. T.; O’Keeffe, M.; Yaghi, O. M. Interwoven Metal-Organic Framework on a Periodic Minimal Surface with Extra-Large Pores. Science 2001, 291, 1021-1023.

(27) Zentner, C. A.; Lai, H. W. H.; Greenfield, J. T.; Wiscons, R. A.; Zeller, M.; Campana, C. F.; Talu, O.; FitzGerald, S. A.; Rowsell, J. L. C. High Surface Area and $Z^{\prime}$ in a Thermally Stable 8-Fold Polycatenated Hydrogen-Bonded Framework. Chem. Commun. 2015, 51, 1164211645 .

(28) Ciesielski, A.; Samorì, P. Supramolecular Assembly/Reassembly Processes: Molecular Motors and Dynamers Operating at Surfaces. Nanoscale 2011, 3, 1397-1410.

(29) Colson, J. W.; Woll, A. R.; Mukherjee, A.; Levendorf, M. P.; Spitler, E. L.; Shields, V. B.; Spencer, M. G.; Park, J.; Dichtel, W. R. Oriented 2D Covalent Organic Framework Thin Films on Single-Layer Graphene. Science 2011, 332, 228-231.

(30) Ruben, M.; Payer, D.; Landa, A.; Comisso, A.; Gattinoni, C.; Lin, N.; Collin, J.-P.; Sauvage, J.-P.; De Vita, A.; Kern, K. 2D Supramolecular Assemblies of Benzene-1,3,5-triyl-tribenzoic Acid: Temperature-Induced Phase Transformations and Hierarchical Organization with Macrocyclic Molecules. J. Am. Chem. Soc. 2006, 128, 15644-15651.

(31) Silly, F. Two-Dimensional 1,3,5-Tris-(4-carboxyphenyl)-benzene Self-Assembly at the 1-Phenyloctane/Graphite Interface Revisited. J. Phvs. Chem. C 2012, 116, 10029-10032.

(32) Lee, S. L.; Fang, Y.; Velpula, G.; Cometto, F. P.; Lingenfelder, M.; Müllen, K.; Mali, K. S.; De Feyter, S. Reversible Local and Global Switching in Multicomponent Supramolecular Networks: Controlled Guest Release and Capture at the Solution/Solid Interface. ACS Nano 2015, 9, 11608-11617.

(33) Li, J.; Gottardi, S.; Solianyk, L.; López, J. C. M.; Stöhr, M. 1,3,5Benzenetribenzoic Acid on $\mathrm{Cu}(111)$ and Graphene $/ \mathrm{Cu}(111)$ : A Comparative STM Study. I. Phys. Chem. C 2016, 120, 18093-18098.

(34) Sing, K. S. W.; Everett, D. H.; Haul, R. A. W.; Moscou, L.; Pierotti, R. A.; Rouquérol, J.; Siemieniewska, T. Reporting Physisorption Data for Gas/Solid Systems with Special Reference to the Determination of Surface Area and Porosity. Pure Appl. Chem. 1985, 57, 603-619.

(35) Barrett, E. P.; Joyner, L. G.; Halenda, P. P. The Determination of Pore Volume and Area Distributions in Porous Substances. I. Computations from Nitrogen Isotherms. J. Am. Chem. Soc. 1951, 73, 373-380.

(36) Sakata, O.; Furukawa, Y.; Goto, S.; Mochizuki, T.; Uruga, T.; Takeshita, K.; Ohashi, H.; Ohata, T.; Matsushita, T.; Takahashi, S.; Tajiri, H.; Ishikawa, T.; Nakamura, M.; Ito, M.; Sumitani, K.; Takahashi, T.; Shimura, T.; Saito, A.; Takahasi, M. Beamline for Surface and Interface Structures at SPring-8. Surf. Rev. Lett. 2003, 10, 543-547. 